\title{
Quadratic Forms for Singular Perturbations of the Laplacian
}

By

\author{
Alessandro TETA*
}

\begin{abstract}
Singular perturbations of $-\Delta$ in $L^{2}\left(R^{3}\right)$ supported by points, regular curves and regular surfaces are considered. Using a renormalization technique the corresponding quadratic forms are constructed and a complete characterization of the domain and the action of the operators is given, together with explicit expressions for the resolvent.
\end{abstract}

\section{§ 1. Introduction}

In recent years some attempts have been devoted to the construction of Schrödinger operators with potentials supported by sets of zero Lebesgue measure. Such hamiltonians are of great importance in applications as models of a variety of physical situations in quantum mechanics, nuclear physics, solid state physics, scattering in disordered media etc. (see e.g. [4] and references therein). Some interesting connections with a model of antenna in classical electrodynamics are stressed in [13].

The existence of such Schrödinger operators can be proved, under some very general conditions on the support of the potential, using different techniques.

A natural framework for justifying the formal manipulations of $\delta$ potentials often employed in the physical literature is non-standard analysis ([1]); standard methods are used in [2], where a singularly perturbed hamiltonian is given by resolvent limit of smooth approximating operators, and in [3], where a construction is obtained studying the non trivial selfadjoint extensions of the Laplacian restricted to smooth functions vanishing on the support of the perturbation.

A more abstract result is given in [14], where the notion of singular bilinear form defining a perturbation of a positive and selfadjoint operator in a Hilbert

Communicated by T. Kawai, May 1, 1989. Revised February 26, 1990.

* S.I.S.S.A., Strada Costiera 11, 34014 Trieste, Italy. 
space is introduced and conditions for the construction of the perturbed operator are given.

Somehow related questions are discussed in [15], where sufficient conditions for the existence of perturbations of a linear differential operator supported by a submanifold of codimension greater than zero are given, and in [16], where the existence and uniqueness of the solution of a Schrödinger type equation with a potential given by a distribution are proved.

It should be emphasized that, except for some special cases like interactions supported by points ([4]) or by a sphere ([5]), using the above mentioned methods it is difficult to get information about the properties of the constructed operator, e.g. locality of the interaction, detailed structure of the domain, spectrum etc.

The aim of this paper is to develop a new approach, based on the theory of quadratic forms, for the description of Schrödinger operators in $L^{2}\left(R^{3}\right)$ with $\delta$-like interactions supported by particular sets of Lebesgue measure zero in $R^{3}$.

More precisely given a set $\mathcal{E} \subset R^{3}$, where $\mathcal{E}$ can be a finite number of points, a regular curve or a regular surface, we define a quadratic form $F_{\mathcal{E}}$ satisfying the following properties: i) it is closed and lower bounded, ii) $D\left(F_{\mathcal{E}}\right) \supseteq D\left(F_{0}\right)$, where $D\left(F_{0}\right)=H^{1}\left(R^{3}\right)$ and $F_{0}(u, u)=\int_{R^{3}}|\nabla u|^{2} d x$, iii) $F_{\mathcal{E}}(u, v)=F_{0}(u, v)$ for any $u \in D\left(F_{0}\right)$ which vanishes in a neighbourhood of $\mathcal{E}$ and for any $v \in D\left(F_{0}\right)$.

When these conditions are satisfied we say that $F_{\mathcal{E}}$ defines a perturbation of the Laplacian supported by $\mathcal{E}$ (cf. definition given in [1], [6]).

Once the quadratic form is defined we provide a complete characterization of the domain and the action of the selfadjoint operator associated to $F_{\mathcal{E}}$ and also an explicit formula for the resolvent.

Moreover it is clear from the construction that the interaction we are defining has a local character in the sense that it is completely specified by an assigned function on $\mathcal{E}$, which is a measure of the strength of the interaction.

In order to illustrate our construction it is convenient to start with the case in which $\mathcal{E}$ is a finite set of points. As it is well known ([4]), the Schrödinger operator $-\Delta_{\alpha, Y}$ with (point) interactions located on the set $Y=\left\{y_{1}, \cdots, y_{N}\right\}$, $y_{i} \in R^{3}$, with strength $\alpha=\left\{\alpha_{1}, \cdots, \alpha_{N}\right\}, \alpha_{i} \in R$, is the unique selfadjoint operator in $L^{2}\left(R^{3}\right)$ with resolvent

$$
\left(-\Delta_{\alpha, Y}+\lambda\right)^{-1}=G^{\lambda}+\sum_{i, j=1}^{N} G^{\lambda}\left(\cdot-y_{i}\right)\left[\Gamma_{\alpha, Y}(\lambda)^{-1}\right]_{i j} G^{\lambda}\left(\cdot-y_{j}\right)
$$

where, for $\lambda>0, \Gamma_{\alpha, Y}(\lambda)$ is the $N \times N$ matrix 


$$
\Gamma_{\alpha, Y}(\lambda)=\left[\left(\alpha_{i}+\frac{\sqrt{\lambda}}{4 \pi}\right) \delta_{i j}-\tilde{G}^{\lambda}\left(y_{i}-y_{j}\right)\right]_{i, j=1}^{N}
$$

and

$$
\begin{gathered}
G^{\lambda}=(-\Delta+\lambda)^{-1} \\
\tilde{G}^{\lambda}(x)=\left\{\begin{array}{lll}
G^{\lambda}(x) & \text { if } & x \neq 0 \\
0 & \text { if } & x=0
\end{array}\right. \\
G^{\lambda}(x)=\frac{e^{-v \bar{\lambda}|x|}}{4 \pi|x|} .
\end{gathered}
$$

The domain of $-\Delta_{\alpha, Y}$, as shown by formula (1.1), contains functions which do not belong to $H^{1}\left(R^{3}\right)$ and have singularities in $y_{1}, \cdots, y_{N}$ of the type $\left|\cdot-y_{i}\right|^{-1}$, $i=1, \cdots, N$.

Using the analogy with electrostatics we interpret this by saying that, for each $u \in D\left(-\Delta_{\alpha, Y}\right)$, one can define $N$ point charges $Q_{u}=\left\{Q_{u}^{1}, \cdots, Q_{u}^{N}\right\}$, depending on the singularity of $u$ in $Y$, such that the potential $u-G_{Y}^{\lambda} Q_{u},\left(G_{Y}^{\lambda} Q_{u}\right.$ is a short notation for $\left.\sum_{i=1}^{N} Q_{u}^{i} G^{\lambda}\left(\cdot-y_{i}\right)\right)$ has finite energy, i.e. belongs to $H^{1}\left(R^{3}\right)$.

This suggests that the quadratic form $F_{\alpha, Y}$, associated to $-\Delta_{\alpha, Y}$, can be decomposed into the sum $F_{\alpha, Y}=\mathscr{F}_{Y}^{\lambda}+\Phi_{\alpha, Y}^{\lambda}$, where $\mathscr{F}_{Y}^{\lambda}$ is essentially the energy associated to the regularized potential $u-G_{Y}^{\lambda} Q_{u}$ and $\Phi_{\alpha, Y}^{\lambda}$ is a sort of renormalized energy of the system of the point charges $Q_{u}$ located in $Y$ (see $\S 2$ ).

The same idea is then applied to the more interesting case of a perturbation of the Laplacian supported by a regular curve $C$; one has only to replace the point charges $Q_{u}$ with a linear charge $\xi_{u}$ distributed on $C$. The resulting quadratic form $F_{\beta, C}$ is essentially the sum of the energy $\mathscr{F}_{C}^{\lambda}$ associated to $u-G_{C}^{\lambda} \xi_{u}$, where $G_{C}^{\lambda} \xi_{u}$ is the potential produced by $\xi_{u}$, and of an extra term $\Phi_{\beta, C}^{\lambda}$ due to the renormalized energy of $\xi_{u}$ (see $\S 3$ ).

We remark that, exploiting further the analysis in [7], perturbations of the Laplacian supported by $Y$ or $C$ can also be obtained as suitable limits of Robin boundary value problems with data on smooth surfaces shrinking to $Y$ or $C$ respectively.

Finally we will briefly consider the well known case of a perturbation supported by a regular surface and we will show, by simple algebraic manipulations, that the corresponding quadratic form can be written in terms of a surface charge, in analogy with the previous cases (see §4).

\section{§ 2. Point Interactions}

Let $Y=\left\{y_{1}, \cdots, y_{N}\right\}$ be a set of $N$ distinct points of $R^{3}$ and $\alpha=\left\{\alpha_{1}, \cdots, \alpha_{N}\right\}$, 
with $\alpha_{i} \in R, i=1, \cdots, N$. For each positive $\lambda$ let us define the following quadratic form in $L^{2}\left(R^{3}\right)$

$$
\begin{gathered}
D\left(F_{\alpha, Y}\right)=\left\{u \in L^{2}\left(R^{3}\right) \mid \exists Q_{u} \in \mathcal{C}^{N} \text { s.t. } u-G_{Y}^{\lambda} Q_{u} \in H^{1}\left(R^{3}\right)\right\} \\
F_{\alpha, Y}(u, u)=\mathscr{F}_{Y}^{\lambda}(u, u)+\Phi_{\alpha, Y}^{\lambda}\left(Q_{u}, Q_{u}\right)
\end{gathered}
$$

where $\mathcal{C}$ is the complex plane and

$$
\begin{gathered}
\mathscr{F}_{Y}^{\lambda}(u, u)=\int_{R^{3}}\left|\nabla\left(u-G_{Y}^{\lambda} Q_{u}\right)\right|^{2} d x+\lambda \int_{R^{3}}\left|u-G_{Y}^{\lambda} Q_{u}\right|^{2} d x-\lambda \int_{R^{3}}|u|^{2} d x \\
\Phi_{\alpha, Y}^{\lambda}\left(Q_{u}, Q_{u}\right)=\sum_{i, j=1}^{N}\left[\Gamma_{\alpha, Y}(\lambda)\right]_{i j} \bar{Q}_{u}^{i} Q_{u}^{j} .
\end{gathered}
$$

It is easy to check that $\Phi_{\alpha, Y}^{\lambda}$ can be obtained as a renormalized energy of the point charges $Q_{u}$

$$
\Phi_{a, Y}^{\lambda}\left(Q_{u}, Q_{u}\right)=-\sum_{i=1}^{N} \bar{Q}_{u}^{i} \lim _{\left|x-y_{i}\right| \rightarrow 0}\left[\sum_{j=1}^{N} Q_{u}^{j} G^{\lambda}\left(x-y_{j}\right)-\frac{Q_{u}^{i}}{4 \pi\left|x-y_{i}\right|}\right]+\sum_{i=1}^{N} \alpha_{i}\left|Q_{u}^{i}\right|^{2} .
$$

Moreover the point charges $Q_{u}^{i}$ corresponding to a given $u \in L^{2}\left(R^{3}\right)$ are uniquely determined; in particular one has $Q_{u}=0$ for any $u \in H^{1}\left(R^{3}\right)$.

In fact the point charges $Q_{u}^{i}$ associated to $u$ can be explicitly computed. Let $B_{r}\left(y_{i}\right)$ be the sphere of radius $r$ centered in $y_{i}$ and $\left|B_{r}\left(y_{i}\right)\right|$ the corresponding volume. For $u \in D\left(F_{\alpha, Y}\right)$, using the Hölder inequality and the continuous embedding of $H^{1}\left(R^{3}\right)$ into $L^{6}\left(R^{3}\right)$, we have

$$
\begin{aligned}
\frac{r}{\left|B_{r}\left(y_{i}\right)\right|} \int_{B_{r}\left(y_{i}\right)}\left|u-G_{Y}^{\lambda} Q_{u}\right| d x & \leq \frac{r}{\left|B_{r}\left(y_{i}\right)\right|^{1 / 6}}\left\|u-G_{Y}^{\lambda} Q_{u}\right\|_{L^{6}\left(R^{3}\right)} \\
& \leq \text { const } r^{1 / 2}\left\|u-G_{Y}^{\lambda} Q_{u}\right\|_{H^{1}\left(R^{3}\right)}
\end{aligned}
$$

Then we conclude

$$
\frac{8 \pi}{3} \lim _{r \rightarrow 0} \frac{r}{\left|B_{r}\left(y_{i}\right)\right|} \int_{B_{r}\left(y_{i}\right)} u d x=\frac{8 \pi}{3} Q_{u}^{i} \lim _{r \rightarrow 0} \frac{r}{\left|B_{r}\left(y_{i}\right)\right|} \int_{B_{r}\left(y_{i}\right)} G^{\lambda}\left(x-y_{i}\right) d x=Q_{u}^{i} .
$$

Remark 1. Notice that the domain $D\left(F_{\alpha, Y}\right)$ is independent of $\lambda>0$. Moreover for any $\lambda, \lambda^{\prime}>0$

$$
\begin{aligned}
& \mathscr{F}_{Y}^{\lambda}(u, u)-\mathscr{F}_{Y}^{\lambda^{\prime}}(u, u) \\
& \left.=\int_{R^{3}} 1-\Delta\left(G_{Y}^{\lambda^{\prime}} Q_{u}-G_{Y}^{\lambda} Q_{u}\right)\right]\left(\bar{u}-G_{Y}^{\lambda^{\prime}} \bar{Q}_{u}\right) d x \\
& \quad+\int_{R^{3}}\left[-\Delta\left(G_{Y}^{\lambda^{\prime}} \bar{Q}_{u}-G_{Y}^{\lambda} \bar{Q}_{u}\right)\right]\left(u-G_{Y}^{\lambda} Q_{u}\right) d x \\
& \quad+\lambda \int_{R^{3}}\left|G_{Y}^{\lambda} Q_{u}\right|^{2} d x-\lambda \int_{R^{3}} \bar{u} G_{Y}^{\lambda} Q_{u} d x-\lambda \int_{R^{3}} u G_{Y}^{\lambda} \bar{Q}_{u} d x
\end{aligned}
$$




$$
\begin{aligned}
& -\lambda^{\prime} \int_{R^{3}}\left|G_{Y}^{\lambda^{\prime}} Q_{u}\right|^{2} d x+\lambda^{\prime} \int_{R^{3}} \bar{u} G_{Y}^{\lambda^{\prime}} Q_{u} d x+\lambda^{\prime} \int_{R^{3}} u G_{Y}^{\lambda^{\prime}} \bar{Q}_{u} d x \\
= & \left(\lambda^{\prime}-\lambda\right) \int_{R^{3}}\left(G_{Y}^{\lambda} Q_{u}\right)\left(G_{Y}^{\lambda^{\prime}} \overline{Q_{u}}\right) d x=\sum_{i, j=1}^{N} \bar{Q}_{u}^{i} Q_{u}^{j}\left[G^{\lambda}\left(y_{i}-y_{j}\right)-G^{\lambda^{\prime}}\left(y_{i}-y_{j}\right)\right] \\
= & -\Phi_{a, Y}^{\lambda}\left(Q_{u}, Q_{u}\right)+\Phi_{a, Y}^{\lambda^{\prime}}\left(Q_{u}, Q_{n}\right)
\end{aligned}
$$

where we have used the equation $-\Delta\left(G_{Y}^{\lambda^{\prime}} Q_{u}-G_{Y}^{\lambda} Q_{u}\right)=\lambda G_{Y}^{\lambda} Q_{u}-\lambda^{\prime} G_{Y}^{\lambda^{\prime}} Q_{u}$, the resolvent identity and an integration by parts. We conclude that $F_{\alpha, Y}$ is in fact independent of the choice of $\lambda>0$; the positive constant $\lambda$ has the only purpose to provide a regularization of the behaviour of the Green's function at infinity.

The following proposition shows that $F_{\alpha, Y}$ defines a perturbation of the Laplacian supported by $Y$.

Proposition 1. $F_{\alpha, Y}$ is a quadratic form in $L^{2}\left(R^{3}\right)$ closed and bounded below. Moreover

$$
\begin{gathered}
D\left(F_{\alpha, Y}\right) \supset D\left(F_{0}\right) \\
F_{\alpha, Y}(u, v)=F_{0}(u, v)
\end{gathered}
$$

for any $u, v \in D\left(F_{0}\right)$.

Proof. The existence of a lower bound is a consequence of the fact that ([4] page 116) $\Gamma_{\alpha, Y}(\lambda)$ is a symmetric matrix whose eigenvalues are all strictly increasing in $\lambda$, so that there exists $\lambda_{0}(\alpha, Y)>0$ such that $\Gamma_{\alpha, Y}(\lambda)$ defines a scalar product in $\mathcal{C}^{N}$ for all $\lambda>\lambda_{0}(\alpha, Y)$. To prove that the form is closed it is more convenient to consider

$$
F_{\alpha, Y}^{\lambda}(u, u)=F_{\alpha, Y}(u, u)+\lambda \int_{R^{3}}|u|^{2} d x
$$

for any $\lambda>\lambda_{0}(\alpha, Y)$. For any sequence $\left\{u_{n}\right\} \subset D\left(F_{a, Y}^{\lambda}\right)$ converging to $u$ in $L^{2}\left(R^{3}\right)$ and such that $\lim _{n, m} F_{\alpha, Y}^{\lambda}\left(u_{n}-u_{m}, u_{n}-u_{m}\right)=0$ one has

$$
\begin{gathered}
\lim _{n, m}\left\|w_{n}-w_{m}\right\|_{H^{1}\left(R^{3}\right)}=0 \\
\lim _{n, m} \Phi_{\alpha, Y}^{\lambda}\left(Q_{u_{n}}-Q_{u_{m}}, Q_{u_{n}}-Q_{u_{m}}\right)=0
\end{gathered}
$$

where $w_{n}=u_{n}-G_{Y}^{\lambda} Q_{u_{n}}$. Then there exist $w \in H^{1}\left(R^{3}\right)$ and $Q \in \mathcal{C}^{N}$ such that

$$
\begin{gathered}
\lim _{n}\left\|w_{n}-w\right\|_{H^{1}\left(R^{3}\right)}=0 \\
\lim _{n}\left\|G_{Y}^{\lambda} Q_{u_{n}}-G_{Y}^{\lambda} Q\right\|_{L^{2}\left(R^{3}\right)}=0 .
\end{gathered}
$$

Formulas (2.10), (2.11) and the uniqueness of the strong limit give $u=$ 
$w+G_{Y}^{\lambda} Q$, i.e. $u \in D\left(F_{\omega, Y}^{\lambda}\right)$, moreover

$$
\lim _{n} F_{a, Y}^{\lambda}\left(u-u_{n}, u-u_{n}\right)=0
$$

and the closedness of $F_{\alpha, Y}$ is proved. Finally the inclusion relation (2.5) and the equality (2.6) can be easily checked.

Q.E.D.

Using the explicit form of $F_{\alpha, Y}$, it is not hard to reconstruct the domain and the action of the associated selfadjoint operator $-\Delta_{\alpha, Y}$

$$
\begin{gathered}
D\left(-\Delta_{\alpha, Y}\right)=\left\{u \in D\left(F_{\alpha, Y}\right) \mid u-G_{Y}^{\lambda} Q_{u} \in H^{2}\left(R^{3}\right),\right. \\
\left.\left(u-G_{Y}^{\lambda} Q_{u}\right)\left(y_{i}\right)=\sum_{j=1}^{N}\left[\Gamma_{\alpha, Y}(\lambda)\right]_{i j} Q_{u}^{j}, \quad i=1, \cdots, N\right\} \\
\left(-\Delta_{\alpha, Y}+\lambda\right) u=(-\Delta+\lambda)\left(u-G_{Y}^{\lambda} Q_{u}\right) .
\end{gathered}
$$

Moreover formula (1.1) for the resolvent can be easily obtained. The proof of (2.13), (2.14), (1.1) can be carried out along the same line as the proof of the propositions 4, 5 of the next section and is omitted here.

Remark 2. If one defines $r_{i}=\left|x-y_{i}\right|, \forall x \in R^{3}, i=1, \cdots, N$, then it can be verified that the boundary condition satisfied by $u \in D\left(-\Delta_{\alpha, Y}\right)$ at the points $y_{1}, \cdots, y_{N}$ can be written in the form extensively used in the literature (see [4], [11])

$$
\lim _{r_{i} \rightarrow 0}\left[\frac{\partial\left(r_{i} u\right)}{\partial r_{i}}-4 \pi \alpha_{i}\left(r_{i} u\right)\right]=0 \quad i=1, \cdots, N .
$$

We finally observe that a perturbation of the Laplacian supported by points in dimension two can be constructed, following exactly the same line as that of the three dimensional case, the only difference being the logarithmic singularity of the two dimensional Green's function for $-\Delta+\lambda$.

\section{§3. Perturbations Supported by Curves}

The construction of a perturbation of the Laplacian supported by a curve is more delicate. The essential reason is that the space of the linear charges distributed on the curve is infinite-dimensional, so that in the definition of the renormalized energy of the linear charge distribution one has to face the problems of domain and closedness.

Let $C$ be a curve in $R^{3}$ of class $C^{1}$ and, for a chosen initial point and orientation, let $y=y(s), s \in I$, be a parametric representation of $C$, where $I$ can be a finite interval (closed curve) or the whole real line (infinite open curve).

Typically $s$ will be the arc-length of $C$ relative to the chosen initial point. 
Moreover we assume that there exists a positive constant $\varepsilon_{0}$ such that the following two conditions are satisfied.

C-1

$$
\left|y(s)-y\left(s^{\prime}\right)\right| \geq\left|s-s^{\prime}\right|\left(1-c\left|s-s^{\prime}\right|^{\tau}\right) \quad \text { whenever }\left|s-s^{\prime}\right| \leq \varepsilon_{0}
$$

where $\tau, c$ are positive constants satisfying $c \varepsilon_{0}^{\tau}<1$.

\section{C-2}

$$
\left|y(s)-y\left(s^{\prime}\right)\right|>\varepsilon_{0}\left(1-c \varepsilon_{0}^{\tau}\right)+K \log \frac{\left|s-s^{\prime}\right|}{\varepsilon_{0}} \text { whenever }\left|s-s^{\prime}\right|>\varepsilon_{0}
$$

where $K$ is a positive constant.

Conditions C-1, C-2 guarantee in particular that the curve cannot have multiple points; moreover one can verify that

$$
\sup _{s \in I} \int_{I} \exp \left(-\sqrt{\lambda}\left|y(s)-y\left(s^{\prime}\right)\right|\right) d s^{\prime}<+\infty \quad \text { for } \quad \lambda>K^{-2} .
$$

In fact, using C-1, C-2, we have

$$
\begin{aligned}
& \int_{I} \exp \left(-\sqrt{\lambda}\left|y(s)-y\left(s^{\prime}\right)\right|\right) d s^{\prime} \leq \int_{\left|s-s^{\prime}\right| \leq \varepsilon_{0}} \exp \left[-\sqrt{\lambda}\left|s-s^{\prime}\right|\left(1-c\left|s-s^{\prime}\right|^{\tau}\right)\right] d s^{\prime} \\
& \quad+\exp \left[-\sqrt{\lambda} \varepsilon_{0}\left(1-c \varepsilon_{0}^{\tau}\right)\right] \varepsilon_{0}^{\sqrt{\lambda} K} \int_{\left|s-s^{\prime}\right|>\varepsilon_{0}} \frac{1}{\left|s-s^{\prime}\right|^{\sqrt{\lambda} K}} d s^{\prime} \\
& \leq 2 \varepsilon_{0} \exp \left(\sqrt{\bar{\lambda}} c \varepsilon_{0}^{1+\tau}\right)\left(1+\frac{\exp \left(-\sqrt{\lambda} \varepsilon_{0}\right)}{\sqrt{\lambda} K-1}\right) .
\end{aligned}
$$

As a preliminary step in the construction of the quadratic form we define

$$
\begin{aligned}
a^{\lambda}(s)= & -\int_{\left|s-s^{\prime}\right|>\varepsilon} G^{\lambda}\left(y(s)-y\left(s^{\prime}\right)\right) d s^{\prime} \\
& +\int_{\left|s-s^{\prime}\right|<\varepsilon}\left[\frac{1}{4 \pi\left|s-s^{\prime}\right|}-G^{\lambda}\left(y(s)-y\left(s^{\prime}\right)\right)\right] d s^{\prime}-\frac{1}{2 \pi} \log 2 \varepsilon
\end{aligned}
$$

for any fixed $\lambda>0$ and $\varepsilon<\varepsilon_{0}$. We observe that $a^{\lambda}(s)$ depends only on the geometry of $C$ and it is a constant in the particular cases of a straight line and of a circumference of radius $\rho$

$$
\begin{gathered}
a_{s . l .}^{\lambda}=\frac{1}{2 \pi}\left(\kappa+\log \frac{\sqrt{\lambda}}{2}\right) \\
a_{\text {circ. }}^{\lambda}=\frac{1}{2 \pi} \int_{0}^{\pi / 2}\left[\frac{1}{t}-\frac{\exp (-2 \sqrt{\lambda} \rho \sin t)}{\sin t}\right] d t-\frac{1}{2 \pi} \log 2 \pi \rho
\end{gathered}
$$


where $\kappa$ is the Euler's constant. Moreover one can verify that $a^{\lambda}(s)$ is in fact independent of the choice of $\varepsilon<\varepsilon_{0}$ and it is a continuous and bounded function of $s \in I$ for any $\lambda>0$. We shall need the following technical lemma.

Lemma 1.

$$
\lim _{\lambda \rightarrow+\infty} \inf _{s \in I} a^{\lambda}(s)=+\infty .
$$

Proof. The first integral in the r.h.s. of (3.2) can be estimated through C-2; one obtains the bound

$$
\int_{\left|s-s^{\prime}\right|>\varepsilon} G^{\lambda}\left(y(s)-y\left(s^{\prime}\right)\right) d s^{\prime}<\frac{1}{4 \pi \varepsilon_{0}\left(1-c \varepsilon_{0}^{\tau}\right)} \sup _{s \in I} \int_{I} \exp \left(-\sqrt{\lambda}\left|y(s)-y\left(s^{\prime}\right)\right|\right) d s^{\prime} .
$$

Moreover using Condition C-1 one has

$$
\begin{aligned}
& \int_{\left|s-s^{\prime}\right|<8}\left[\frac{1}{4 \pi\left|s-s^{\prime}\right|}-G^{\lambda}\left(y(s)-y\left(s^{\prime}\right)\right)\right] d s^{\prime} \\
& \quad \geq \int_{\left|s-s^{\prime}\right|<\varepsilon} \frac{1}{4 \pi\left|s-s^{\prime}\right|}\left\{1-\frac{\exp \left[-\sqrt{\lambda}\left|s-s^{\prime}\right|\left(1-c\left|s-s^{\prime}\right|^{\tau}\right)\right]}{1-c\left|s-s^{\prime}\right|^{\tau}}\right\} d s^{\prime} \\
& \quad \equiv \frac{1}{4 \pi} J(\sqrt{\lambda}) .
\end{aligned}
$$

For $\lambda$ sufficiently large, the last integral is positive and satisfies

$$
\frac{d J(\sqrt{\lambda})}{d \sqrt{\lambda}}=2 \int_{0}^{e} \exp \left[-\sqrt{\lambda} \zeta\left(1-c \zeta^{\tau}\right)\right] d \zeta>\frac{1}{\sqrt{\lambda}} .
$$

The proof of the lemma then follows from (3.1), (3.4) and (3.6).

Q.E.D.

We now introduce a class of admissible linear charges distributed on $C$. For a given continuous, bounded from below and real valued function $\beta$ defined on $C$ and for each positive number $\lambda$ let us define

$$
\begin{gathered}
D\left(\Phi_{\beta, c}^{\lambda}\right)=\left\{\xi \in L^{2}(I) \mid \Phi_{\beta, C}^{\lambda}(\xi, \xi)<+\infty\right\} \\
\Phi_{\beta, c}^{\lambda}(\xi, \xi)=\frac{1}{2} \int_{I \times I}\left|\xi(s)-\xi\left(s^{\prime}\right)\right|^{2} G^{\lambda}\left(y(s)-y\left(s^{\prime}\right)\right) d s d s^{\prime} \\
+\int_{I}|\xi(s)|^{2}\left(a^{\lambda}(s)+\beta(s)\right) d s .
\end{gathered}
$$

Proposition 2. There exists $\lambda_{0}(\beta, C)>0$ such that, for any $\lambda>\lambda_{0}(\beta, C)$, $D\left(\Phi_{\beta, c}^{\lambda}\right)$ is a Hilbert space w.r. to the scalar product $\Phi_{\beta, c}^{\lambda}(\cdot, \cdot)$.

Proof. The existence of a $\lambda_{0}(\beta, C)>0$ such that $\Phi_{\beta, c}^{\lambda}\left(\cdot,{ }^{\circ}\right)$ defines a positive definite scalar product for $\lambda>\lambda_{0}(\beta, C)$ is a consequence of Lemma 1 . In 
order to prove the completeness one has only to mimic the Riesz-Fisher proof of the completeness of $L^{2}$. Given a Cauchy sequence $\left\{\xi_{n}\right\}$ in $D\left(\Phi_{\beta, C}^{\lambda}\right)$, it is sufficient to prove convergence for a subsequence. Pick a subsequence, still denoted by $\left\{\xi_{n}\right\}$, such that $\Phi_{\beta, c}^{\lambda}\left(\xi_{n}-\xi_{n+1}, \xi_{n}-\xi_{n+1}\right)<2^{-n}$. Then

$$
\Theta(s)=\sum_{n=1}^{\infty}\left|\xi_{n}(s)-\xi_{n+1}(s)\right|
$$

is the monotone limit of

$$
\Theta_{N}(s)=\sum_{n=1}^{N}\left|\xi_{n}(s)-\xi_{n+1}(s)\right|
$$

and by the monotone convergence theorem $\Theta \in L^{2}(I)$ and $\Theta<+\infty$ a.e. Moreover $\left|\Theta_{N}(s)-\Theta_{N}\left(s^{\prime}\right)\right|^{2} G^{\lambda}\left(y(s)-y\left(s^{\prime}\right)\right)$ converges a.e. in $I \times I$ to $\mid \Theta(s)-$ $\left.\Theta\left(s^{\prime}\right)\right|^{2} G^{\lambda}\left(y(s)-y\left(s^{\prime}\right)\right)$ and

$$
\Phi_{\beta, C}^{\lambda}\left(\Theta_{N}, \Theta_{N}\right) \leq \sum_{n=1}^{N} \Phi_{\beta, C}^{\lambda}\left(\left|\xi_{n+1}-\xi_{n}\right|,\left|\xi_{n+1}-\xi_{n}\right|\right)<\text { const }
$$

so by the Fatou lemma we get $\Theta \in D\left(\Phi_{\beta, C}^{\lambda}\right)$. Thus $\xi_{1}+\sum_{n=1}^{\infty}\left(\xi_{n+1}-\xi_{n}\right)$ is absolutely convergent to a $\operatorname{sum} \xi$ with $\left|\xi-\xi_{1}\right| \leq \Theta$, so that $\xi \in D\left(\Phi_{\beta, C}^{\lambda}\right)$. It is now straightforward to show that $\lim _{n} \Phi_{\beta, C}^{\lambda}\left(\xi-\xi_{n}, \xi-\xi_{n}\right)=0$.

Q.E.D.

By Proposition 2 we get that $\Phi_{\beta, C}^{\lambda}$ is, for $\lambda>\lambda_{0}(\beta, C)$, a positive and closed quadratic form in $L^{2}(I)$ so it defines a positive and selfadjoint operator $\Gamma_{\beta, c}(\lambda)$, which acts on smooth $\xi$ as follows

$$
\left[\Gamma_{\beta, c}(\lambda) \xi\right](s)=\int_{I}\left[\xi(s)-\xi\left(s^{\prime}\right)\right] G^{\lambda}\left(y(s)-y\left(s^{\prime}\right)\right) d s^{\prime}+\xi(s)\left[\beta(s)+a^{\lambda}(s)\right] .
$$

Now we want to recall two useful properties of the potential

$$
G_{C}^{\lambda} \xi(x)=\int_{I} \xi(s) G^{\lambda}(x-y(s)) d s \quad x \in R^{3}
$$

produced by the linear charge $\xi \in D\left(\Phi_{\beta, C}^{\lambda}\right)$. We observe first that the map $\xi \mapsto G_{C}^{\lambda} \xi, \lambda>\lambda_{0}(\beta, C)$, is a linear bounded map from $D\left(\Phi_{\beta, C}^{\lambda}\right)$ to $L^{2}\left(R^{3}\right)$, and its norm converges to zero for $\lambda \rightarrow+\infty$. The proof, based on an application of the Fubini theorem, is straightforward. Next it can be shown that $G_{C}^{\lambda} \xi \notin H^{1}\left(R^{3}\right)$. We give here a sketch of the proof. The regularity conditions C-1, C-2 allow us to define, for any $\delta>0$ sufficiently small, a neighborhood of $C$

$$
\begin{aligned}
C_{\delta}= & \left\{x \in R^{3} \mid \exists ! y\left(s_{x}\right) \in C \text { s.t. } x \text { lies on the normal plane to } C\right. \\
& \text { in } \left.y\left(s_{x}\right) \text { and }\left|x-y\left(s_{x}\right)\right|<\delta\right\} .
\end{aligned}
$$

Then an integration by parts yields 


$$
\begin{aligned}
\int_{R^{3} \backslash C_{\delta}}\left|\nabla G_{C}^{\lambda} \xi\right|^{2} d x+\lambda \int_{R^{3} \backslash C_{\delta}} G_{C}^{\lambda}\left(|\xi|^{2}\right) G_{C}^{\lambda} 1 d x \\
=-\frac{1}{2} \int_{I \times I} d s d s^{\prime}\left|\xi(s)-\xi\left(s^{\prime}\right)\right|^{2} \int_{R^{3} \backslash C_{\delta}} d x \nabla_{x} G^{\lambda}(x-y(s)) \cdot \nabla_{x} G^{\lambda}\left(x-y\left(s^{\prime}\right)\right) \\
\quad+\int_{\partial C_{\delta}}\left(G_{C}^{\lambda} 1-\frac{1}{2 \pi} \log \frac{1}{\delta}\right) \frac{\partial G_{C}^{\lambda}\left(|\xi|^{2}\right)}{\partial n} d \Sigma+\frac{1}{2 \pi} \log \frac{1}{\delta} \int_{\partial C_{\delta}} \frac{\partial G_{C}^{\lambda}\left(|\xi|^{2}\right)}{\partial n} d \Sigma
\end{aligned}
$$

where $G_{C}^{\lambda} 1(x)=\int_{I} G^{\lambda}(x-y(s)) d s, x \in R^{3}$. The second term in the 1.h.s. of (3.12) remains bounded in the limit $\delta \rightarrow 0$ and the first two terms in the r.h.s. of (3.12) converge to $-\Phi_{\beta, c}^{\lambda}(\xi, \xi)+\int_{I}|\xi(s)|^{2} \beta(s) d s$, which is finite by hypothesis.

Concerning the integral in the last term of (3.12) we have

$$
\begin{aligned}
\int_{\partial c_{\delta}} \frac{\partial G^{\lambda}\left(|\xi|^{2}\right)}{\partial n} d \Sigma & =\int_{R^{3} \backslash C_{\delta}} \Delta G^{\lambda}\left(|\xi|^{2}\right) d x=\lambda \int_{R^{3} \backslash C_{\delta}} G^{\lambda}\left(|\xi|^{2}\right) d x \\
& =\lambda \int_{I} d s|\xi(s)|^{2} \int_{R^{3} \backslash C_{\delta}} d x G^{\lambda}(x-y(s)) \leq \int_{I}|\xi(s)|^{2} d s .
\end{aligned}
$$

It is now evident that the last term in (3.12) diverges logarithmically for $\delta \rightarrow 0$ and then our assertion is proved.

The above procedure shows, in particular, that $\Phi_{\beta, c}^{\lambda}(\xi, \xi)$ can be considered as a renormalized energy of the linear charge distribution (cf. $\S 1$ ). Now we have all the ingredients to define, for $\lambda>\lambda_{0}(\beta, C)$, the following quadratic form in $L^{2}\left(R^{3}\right)$

$$
\begin{gathered}
D\left(F_{\beta, C}\right)=\left\{u \in L^{2}\left(R^{3}\right) \mid \exists \xi_{u} \in D\left(\Phi_{\beta, C}^{\lambda}\right) \text { s.t. } u-G_{C}^{\lambda} \xi_{u} \in H^{1}\left(R^{3}\right)\right\} \\
F_{\beta, C}(u, u)=\mathscr{F}_{c}^{\lambda}(u, u)+\Phi_{\beta, C}^{\lambda}\left(\xi_{u}, \xi_{u}\right)
\end{gathered}
$$

where

$$
\mathscr{F}_{C}^{\lambda}(u, u)=\int_{R^{3}}\left|\nabla\left(u-G_{C}^{\lambda} \xi_{u}\right)\right|^{2} d x+\lambda \int_{R^{3}}\left|u-G_{C}^{\lambda} \xi_{u}\right|^{2} d x-\lambda \int_{R^{3}}|u|^{2} d x .
$$

Given $u \in L^{2}\left(R^{3}\right)$ then the corresponding linear charge $\xi_{u}$ is uniquely determined.

In fact if there were $\xi_{u}, \xi_{u}^{\prime} \in D\left(\Phi_{\beta, C}^{\lambda}\right)$ such that $u-G_{C}^{\lambda} \xi_{u} \in H^{1}\left(R^{3}\right)$ and $u-G_{C}^{\lambda} \xi_{u}^{\prime} \in H^{1}\left(R^{3}\right)$ we would also have $G_{C}^{\lambda}\left(\xi_{u}-\xi_{u}^{\prime}\right) \in H^{1}\left(R^{3}\right)$ which is absurd, by the above considerations, unless $\xi_{u}=\xi_{u}^{\prime}$.

In particular this means that $\xi_{u}=0$ for $u \in H^{1}\left(R^{3}\right)$.

Moreover from reasoning as in Remark 1 it is not hard to show that $F_{\beta, C}$ is in fact independent of the choice of $\lambda>0$. The following proposition shows that $F_{\beta, C}$ defines a perturbation of the Laplacian supported by $C$. 
Proposition 3. $\quad F_{\beta, C}$ is a closed quadratic form in $L^{2}\left(R^{3}\right)$ bounded below; one has

$$
\begin{gathered}
D\left(F_{\beta, c}\right) \supset D\left(F_{0}\right) \\
F_{\beta, c}(u, v)=F_{0}(u, v)
\end{gathered}
$$

for any $u, v \in D\left(F_{0}\right)$.

Proof. The existence of a lower bound for $F_{\beta, C}$ is a consequence of the positivity of $\Phi_{\beta, C}^{\lambda}$ for $\lambda>\lambda_{0}(\beta, C)$. In order to prove that it is closed we consider

$$
F_{\beta, c}^{\lambda}(u, u)=F_{\beta, c}(u, u)+\lambda \int_{R^{3}}|u|^{2} d x
$$

for any $\lambda>\lambda_{0}(\beta, C)$. For any sequence $\left\{u_{n}\right\} \subset D\left(F_{\beta, C}^{\lambda}\right)$ converging to $u$ in $L^{2}\left(R^{3}\right)$ and such that $\lim _{n, m} F_{\beta, c}^{\lambda}\left(u_{n}-u_{m}, u_{n}-u_{m}\right)=0$, one has

$$
\begin{gathered}
\lim _{n, m}\left\|z_{n}-z_{m}\right\|_{H^{1}\left(R^{3}\right)}=0 \\
\lim _{n, m} \Phi_{\beta, c}^{\lambda}\left(\xi_{u_{n}}-\xi_{u_{m}}, \xi_{u_{n}}-\xi_{u_{m}}\right)=0
\end{gathered}
$$

where $z_{n}=u_{n}-G_{C}^{\lambda} \xi_{u_{n}}$. Thus there exist $z \in H^{1}\left(R^{3}\right)$ and $\xi \in D\left(\Phi_{\beta, C}^{\lambda}\right)$ such that

$$
\begin{gathered}
\lim _{n}\left\|z_{n}-z\right\|_{H^{1}\left(R^{3}\right)}=0 \\
\lim _{n} \Phi_{\beta, C}^{\lambda}\left(\xi-\xi_{u_{n}}, \xi-\xi_{u_{n}}\right)=0 .
\end{gathered}
$$

By the continuity of the potential of a linear charge and the uniqueness of the strong limit we get $u=z+G_{C}^{\lambda} \xi$, which means $u \in D\left(F_{\beta, C}^{\lambda}\right)$. Moreover by (3.21), (3.22) we have $\lim _{n} F_{\beta, c}^{\lambda}\left(u-u_{n}, u-u_{n}\right)=0$ and the closedness of $F_{\beta, c}^{\lambda}$ is proved. Finally the inclusion relation (3.16) and the equality (3.17) can be easily verified.

Q.E.D.

The selfadjoint operator $-\Delta_{\beta, C}$ associated to $F_{\beta, C}$ is by definition the Schrödinger operator with $\delta$-interaction supported by $C$ of strength $\beta$; its domain and action are completely characterized as follows:

\section{Proposition 4.}

$$
\begin{aligned}
D\left(-\Delta_{\beta, C}\right)= & \left\{u \in D\left(F_{\beta, C}\right) \mid \xi_{u} \in D\left(\Gamma_{\beta, c}(\lambda)\right), u-G_{C}^{\lambda} \xi_{u} \in H^{2}\left(R^{3}\right),\right. \\
& \left.\left(u-G_{C}^{\lambda} \xi_{u}\right)(y(s))=\left(\Gamma_{\beta, C}(\lambda) \xi_{u}\right)(s) \forall s \in I\right\} \\
& \left(-\Delta_{\beta, C}+\lambda\right) u=(-\Delta+\lambda)\left(u-G_{C}^{\lambda} \xi_{u}\right) .
\end{aligned}
$$

Proof. Let $u \in D\left(-\Delta_{\beta, c}\right)$. Then, by definition, there exists $g \in L^{2}\left(R^{3}\right)$ such that 


$$
F_{\beta, C}(u, v)=(v, g)_{L^{2}\left(R^{3}\right)}
$$

for any $v \in D\left(F_{\beta, C}\right)$. In particular for $v \in H^{1}\left(R^{3}\right)$ one has $\xi_{v}=0$ and (3.25) becomes

$$
\int_{R^{3}} \nabla \bar{v} \cdot \nabla\left(u-G_{C}^{\lambda} \xi_{u}\right) d x+\lambda \int_{R^{3}} \bar{v}\left(u-G_{C}^{\lambda} \xi_{u}\right) d x=\int_{R^{3}} \bar{v}(g+\lambda u) d x
$$

which gives $u-G_{C}^{\lambda} \xi_{u} \in H^{2}\left(R^{3}\right)$. Thus an integration by parts yields

$$
(-\Delta+\lambda)\left(u-G_{C}^{\lambda} \xi_{u}\right)=\left(-\Delta_{\beta, c}+\lambda\right) u .
$$

For an arbitrary $v \in D\left(F_{\beta, c}\right)$ equality (3.25) can be written as

$$
\int_{I} \bar{\xi}_{v}(s)\left(u-G_{C}^{\lambda} \xi_{u}\right)(y(s)) d s=\Phi_{\beta, C}^{\lambda}\left(\xi_{v}, \xi_{u}\right)
$$

where (3.26), (3.27) have been used and the order of integration has been interchanged. Equation (3.28) gives now $\xi_{u} \in D\left(\Gamma_{\beta, c}(\lambda)\right)$ and $\left(\Gamma_{\beta, c}(\lambda) \xi_{u}\right)(s)=$ $\left(u-G_{C}^{\lambda} \xi_{u}\right)(y(s))$. Conversely given $u$ belonging to the r.h.s. of (3.23) it is easily checked, following the same line of reasoning, that $u \in D\left(-\Delta_{\beta, C}\right)$ proving (3.24).

Q.E.D.

Remark 3. For $u \in D\left(-\Delta_{\beta, c}\right)$, with a smooth $\xi_{u}$, let us denote by $\hat{u}(y(s), \delta)$ the mean value of $u$ over the circle of (a sufficiently small) radius $\delta$, centered in $y(s) \in C$ and orthogonal to $C$ in $y(s)$; then one can define two continuous function on $C$

$$
\begin{gathered}
\phi_{0}(s)=2 \pi \lim _{\delta \rightarrow 0} \frac{u(y(s), \delta)}{\log 1 / \delta} \\
\phi_{1}(s)=\lim _{\delta \rightarrow 0}\left[u(y(s), \delta)-\frac{\phi_{0}(s)}{2 \pi} \log \frac{1}{\delta}\right]
\end{gathered}
$$

and it can be verified that the boundary condition $\left(u-G_{C}^{\lambda} \xi_{u}\right)(y(s))=\left(\Gamma_{\beta, c}(\lambda) \xi_{u}\right)(s)$ is equivalent to

$$
\phi_{1}(s)=\alpha(s) \phi_{0}(s) .
$$

We remark that the last equation, for a fixed $s \in I$, essentially coincides with the boundary condition defining a point interaction in dimension two (see [4] page 98).

It is also possible to give explicitly the resolvent of $-\Delta_{\beta, C}$

Proposition 5. For $\lambda>\lambda_{0}(\beta, C)$ and $g \in L^{2}\left(R^{3}\right)$ we have 


$$
\left(-\Delta_{\beta, C}+\lambda\right)^{-1} g=G^{\lambda} g+\int_{I}\left[\Gamma_{\beta, c}(\lambda)^{-1}\left(\left.G^{\lambda} g\right|_{c}\right)\right](s) G^{\lambda}(\cdot-y(s)) d s
$$

where $\left.G^{\lambda} g\right|_{c}$ indicates the restriction of $G^{\lambda} g$ to the curve $C$.

Proof. The r.h.s. of (3.32) defines a bounded linear operator from $L^{2}\left(R^{3}\right)$ onto $D\left(-\Delta_{\beta, C}\right)$; using (3.24) one immediately proves the proposition. Q.E.D.

In conclusion we observe that, starting from (3.32), one can investigate spectral properties of $-\Delta_{\beta, c}$ (such as the location of the point spectrum and the absence of the singular continuous spectrum) and study the scattering theory for the pair $\left(-\Delta_{\beta, c},-\Delta\right)$. We plane to come back to these questions in further work.

\section{§4. Perturbations Supported by Surfaces}

It is well known that a perturbation of the Laplacian supported by a regular closed surface $S$ can be defined by the following quadratic form

$$
\begin{gathered}
D\left(F_{\gamma, S}\right)=H^{1}\left(R^{3}\right) \\
F_{\gamma, S}(u, u)=\int_{R^{3}}|\nabla u|^{2} d x+\int_{S} \gamma\left|u_{S}\right|^{2} d \Sigma
\end{gathered}
$$

where $r$ is a smooth, real valued function defined on $S$ and $u_{S}$ denotes the trace of $u$ on $S$. If one defines the surface charge $\sigma_{u}$ associated to $u \in H^{1}\left(R^{3}\right)$

$$
\sigma_{u}=-r u_{s}
$$

and, for any $\lambda>0$, the potential produced by $\sigma_{u}$

$$
G_{S}^{\lambda} \sigma_{u}(x)=\int_{S} \sigma_{u}(\zeta) G^{\lambda}(x-\zeta) d \Sigma(\zeta) \quad x \in R^{3}
$$

then a simple calculation shows that $F_{\gamma, s}$ can be written in a form analogous to the previous cases

$$
F_{\gamma, s}(u, u)=\mathscr{F}_{S}^{\lambda}(u, u)+\Phi_{\gamma, s}^{\lambda}\left(\sigma_{u}, \sigma_{u}\right)
$$

where

$$
\begin{gathered}
\mathscr{F}_{S}^{\lambda}(u, u)=\int_{R^{3}}\left|\nabla\left(u-G_{S}^{\lambda} \sigma_{u}\right)\right|^{2} d x+\lambda \int_{R^{3}}\left|u-G_{S}^{\lambda} \sigma_{u}\right|^{2} d x-\lambda \int_{R^{3}}|u|^{2} d x \\
\Phi_{\gamma, S}^{\lambda}\left(\sigma_{u}, \sigma_{u}\right)=-\int_{S} \frac{\left|\sigma_{u}\right|^{2}}{r} d \Sigma-\int_{S} \bar{\sigma}_{u}\left(G_{S}^{\lambda} \sigma_{u}\right) d \Sigma .
\end{gathered}
$$

$\Phi_{\gamma, s}^{\lambda}$ is clearly a bounded quadratic form in $L^{2}(\Sigma)$, thus it defines a bounded 
selfadjoint operator $\Gamma_{\gamma, s}(\lambda)$ in $L^{2}(\Sigma)$. Using the methods developed in $\S 2$, $\$ 3$ one can reconstruct the domain and the action of the selfadjoint operator $-\Delta_{\gamma, S}$ defined by $F_{\gamma, S}$ and one can calculate the resolvent. The results are summarized below

$$
\begin{gathered}
D\left(-\Delta_{\gamma, S}\right)=\left\{u \in H^{1}\left(R^{3}\right)\left|u-G_{S}^{\lambda} \sigma_{u} \in H^{2}\left(R^{3}\right),\left(u-G_{S}^{\lambda} \sigma_{u}\right)\right|_{s}=\Gamma_{\gamma, S}(\lambda) \sigma_{u}\right\} \\
\left(-\Delta_{\gamma, S}+\lambda\right) u=(-\Delta+\lambda)\left(u-G_{S}^{\lambda} \sigma_{u}\right) \\
\left(-\Delta_{\gamma, S}+\lambda\right)^{-1} g=G^{\lambda} g+\int_{S}\left[\mid \Gamma_{\gamma, S}(\lambda)^{-1}\left(\left.G^{\lambda} g\right|_{s}\right)\right](\zeta) G^{\lambda}(\cdot-\zeta) d \Sigma(\zeta)
\end{gathered}
$$

for any $g \in L^{2}\left(R^{3}\right)$. It is a simple exercise to show that, when $S$ is a sphere, (4.10) reduces to the resolvent given in [5].

\section{Acknowledgments}

I would like to thank Prof. G.F. Dell'Antonio, under whose guidance this work has been undertaken. I am also grateful to Prof. G. Dal Maso, Prof. R. Figari and Dr. M. Balzano for helpful discussions.

\section{References}

[1] Albeverio, S., Fenstad, J.E., Hoegh-Krohn, R. and Lindström, T., Non standard Methods in Stochastic Analysis and Mathematical Physics, Academic Press, New YorkSan Francisco-London, 1986.

[2] Albeverio, S., Fenstad, J.E., Hфegh-Krohn, R., Karwowski, W. and Lindström, T., Schrödinger operators with potentials supported by null sets, preprint in preparation.

[3] Brasche, J.F., Perturbations of self-adjoint operators suppoted by null sets, Dissertation zur Erlangung des Doktorgrades der Fakultät für Mathematik der Universität Bielefeld, 1988.

[4] Albeverio, S., Gesztesy, F., Hфegh-Krohn, R. and Holden, H., Solvable Models in Quantum Mechanics, Springer-Verlag, Berlin-Heidelberg-New York, 1988.

[5] Antoine, J.P., Gesztesy, F. and Shabani, J., Exactly solvable models of sphere interactions in quantum mechanics, J. Phys., A20 (1987), 3687-3712.

[6] Albeverio, S., Fenstad, J.E., Hфegh-Krohn, R., Karwowski, W. and Lindström, T., Perturbations of the Laplacian supported by null sets, with applications to polymer measure and quantum fields, Phys. Lett., 104A (1984), 396-400.

[7] Figari, R., Holden, H. and Teta, A., A law of large numbers and a central limit theorem for the Schrödinger operator with zero-range potentials, J. Stat. Phys., 51 (1988), 205-214.

[8] Kato, T., Perturbation Theory for Linear Operators, Springer-Verlag, Berlin-Heidelberg-New York, 1980.

[9] Simon, B., Quantum Mechanics for Hamiltonian Defined as Quadratic Forms, Princeton U.P., Princeton, NJ, 1971.

[10] Reed, M. and Simon, B., Methods of Modern Mathematical Physics. II. Fourier Analysis, Self-Adjointness, Academic Press, New York-San Francisco-London, 1975. 
[11] Thomas, L.E., Birman-Schwinger bounds for the Laplacian with point interactions, $J$. Math. Phys., 20 (1979), 1848-1850.

[12] Kellogg, O.D., Foundations of Potential Theory, Springer-Verlag, Berlin, 1929.

[13] Grossmann, A. and Wu, T.T., A class of potentials with extremely narrow resonances, Chin. J. Phys., 25 (1987), 129-139.

[14] Koshmanenko, V.D., Singular perturbations defined by forms, BiBoS preprint Nr. $314 / 88$.

[15] Svendsen, E.C., The effect of submanifolds upon essential self-adjointness and deficiency indices", J. Math. Anal. Appl., 80 (1981), 551-565.

[16] Rozanov, Yu. A., On the Schrödinger type equation with generalized potential, Math. USSR Sbornik, 55 (1986), 475-484. 
